\title{
Pasireotide (SOM230) shows efficacy and tolerability in the treatment of patients with advanced neuroendocrine tumors refractory or resistant to octreotide LAR: results from a phase II study
}

\author{
Larry K Kvols, Kjell E Oberg ${ }^{1}$, Thomas M O'Dorisio², Pharis Mohideen ${ }^{3}$, \\ Wouter W de Herder ${ }^{4}$, Rudolf Arnold ${ }^{5}$, Ke Hü ${ }^{3}$, Yilong Zhang ${ }^{3}$, \\ Gareth Hughes ${ }^{6}$, Lowell Anthony ${ }^{7}$ and Bertram Wiedenmann ${ }^{8}$

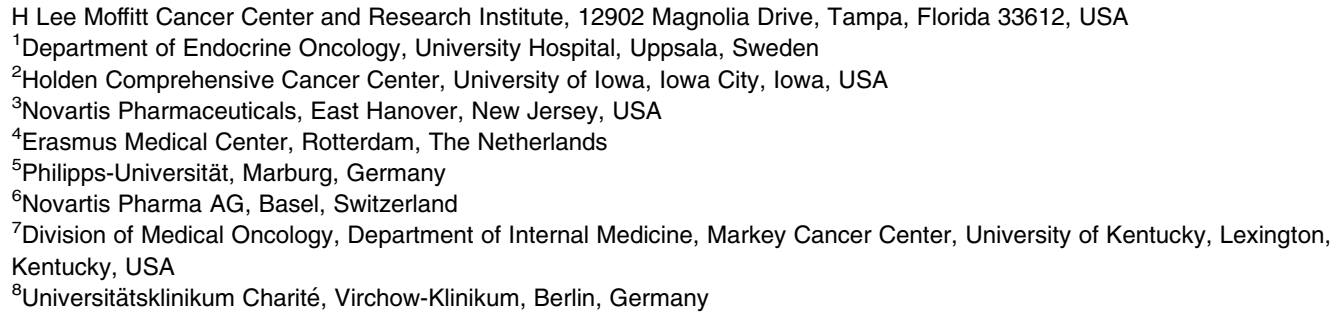

\begin{abstract}
Pasireotide (SOM230) is a novel multireceptor-targeted somatostatin (sst) analog with high binding affinity for sst receptor subtype 1,2, $3\left(s_{1,2,3}\right)$ and $s_{1} t_{5}$. Because of this binding profile, pasireotide may offer symptom control in patients with neuroendocrine tumors (NETs) and carcinoid syndrome no longer responsive to octreotide LAR. This was a phase II, open-label, multicenter study of pasireotide in patients with advanced NET whose symptoms of carcinoid syndrome (diarrhea/flushing) were inadequately controlled by octreotide LAR. Patients received s.c. pasireotide $150 \mu \mathrm{g}$ twice daily (bid), escalated to a maximum dose of $1200 \mu \mathrm{g}$ bid until a clinical response was achieved. Forty-four patients were evaluated for efficacy and 45 for tolerability. Pasireotide $600-900 \mu \mathrm{g}$ s.c. bid effectively controlled the symptoms of diarrhea and flushing in $27 \%$ of patients. Evaluation of tumor response in 23 patients showed 13 with stable disease and ten with progressive disease at study end. The most common drug-related adverse events were nausea $(27 \%)$, abdominal pain $(20 \%)$, weight loss $(20 \%)$, and hyperglycemia $(16 \%)$ and most were of mild or moderate severity. Pasireotide $600-900 \mu \mathrm{g}$ s.c. bid was effective and generally well tolerated in controlling the symptoms of carcinoid syndrome in $27 \%$ of patients with advanced NET refractory or resistant to octreotide LAR therapy.
\end{abstract}

Endocrine-Related Cancer (2012) 19 657-666

\section{Introduction}

Neuroendocrine tumors (NETs) are often asymptomatic and by the time of diagnosis have frequently metastasized, usually to the liver (Modlin et al. 2005). The majority of NETs are gastrointestinal (historically referred to as carcinoid tumors) and are characterized by hypersecretion of biogenic amines, peptides, and polypeptides, such as serotonin, tachykinins, and chromogranin A (CgA; Long et al. 1981, Fajans \& Vinik 1989, Modlin et al. 2005). The excessive release 
of these amines and peptide hormones, especially serotonin and substance $\mathrm{P}$, is responsible for the principal symptoms of diarrhea and flushing associated with carcinoid syndrome.

Surgery is essential in the management of metastatic NETs and can be curative for early disease (Loftus \& van Heerden 1995, Plockinger et al. 2004). However, the majority of patients require medical treatment either as an adjuvant to surgery, particularly when total resection is not possible, or as first-line treatment in patients not indicated for surgery.

The somatostatin (sst) analogs octreotide and lanreotide have been the mainstay for symptom management in patients with NETs. Octreotide and lanreotide have a high affinity for the sst receptor subtype $2\left(\mathrm{sst}_{2}\right)$ and a modest affinity for $\mathrm{sst}_{5}$ (Bruns et al. 1994). However, expression of multiple sst subtypes is observed in NETs (Hofland \& Lamberts 2003). Although octreotide LAR therapy effectively reduces the symptoms of carcinoid syndrome in the majority of patients (Rubin et al. 1999, Modlin et al. 2006), a considerable number of patients will experience an escape from response within months to several years of treatment, whereupon symptoms may return, and the prognosis for these patients is poor (Hofland et al. 2005). Mechanisms by which this desensitization to currently available sst analogs may occur include $\mathrm{sst}_{2}$ internalization and downregulation on tumor cells, over-expression of other sst receptor subtypes (Ronga et al. 1995, Li et al. 2004, Schmid \& Schoeffter 2004), or other unknown mechanisms. Targeting multiple ssts in NETs may provide an effective treatment for the relief of symptoms in patients who are no longer responsive to the currently available sst analogs.

Pasireotide (SOM230) is a novel multireceptortargeted sst analog with high binding affinity to four of the five known sst receptor subtypes $\left(\mathrm{sst}_{1,2,3}\right.$ and $\mathrm{sst}_{5}$; Bruns et al. 2002, Boerlin et al. 2003, Schmid \&
Schoeffter 2004). Compared with octreotide, pasireotide has 30-, 5-, and 39-fold higher affinity for $\mathrm{sst}_{1,3}$ and $\mathrm{sst}_{5}$, respectively, and a slightly lower affinity for $\mathrm{sst}_{2}$ (Bruns et al. 2002, Boerlin et al. 2003, Schmid \& Schoeffter 2004). Because of this receptor binding profile, pasireotide may offer symptom reduction in patients who are inadequately controlled with octreotide LAR therapy. This paper presents results from a phase II, open-label, multicenter study designed to assess the efficacy and safety of pasireotide in patients with advanced NETs whose symptoms were refractory or resistant to octreotide LAR therapy.

\section{Materials and methods}

\section{Study population}

Eligible patients were required to be aged $\geq 18$ years with histopathologically and biochemically confirmed metastatic NETs of the digestive system of any grade with or without disease progression. Patients had elevated urinary 5-hydroxyindole acetic acid (5-HIAA) and/or serum CgA levels, one or more measurable lesions (excluding bone) that demonstrated progression since radiation or had not been previously irradiated, and a Karnofsky performance status $\geq 60$. Inadequate control of disease was defined as a mean of $\geq 4$ bowel movements per day or a mean of $\geq 2$ episodes of flushing per day for at least 2 weeks during the 28-day period following the last dose of octreotide LAR (screening/baseline period). Patients then underwent a 28-day washout period during which time treatment with protocol-approved medication, including octreotide s.c. or over-the-counter medication to control the symptoms of carcinoid syndrome, was permitted up to $48 \mathrm{~h}$ before initial administration of pasireotide (Fig. 1).

Key exclusion criteria included patients with uncontrolled diabetes mellitus, malabsorption

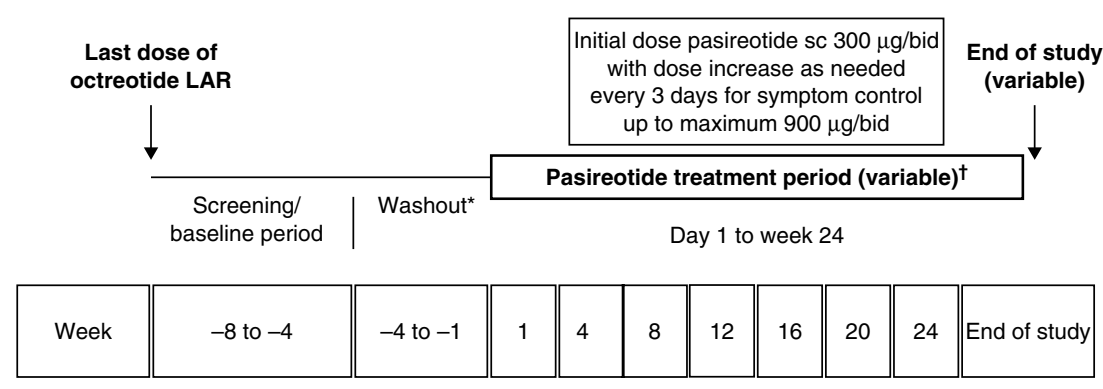

Figure 1 Study design. * Octreotide s.c. could be administered for symptom control up to 2 days before pasireotide initiation. ${ }^{\dagger}$ During pasireotide therapy patients were discouraged from taking over-the-counter diarrhea medications. 
syndrome, short bowel or chologenic diarrhea not controlled by medication, surgery for loco-regional metastasis within 3 months, or any other major surgery within 1 month of study entry. Patients who had received radiolabeled sst analog therapy within 6 months before receiving pasireotide, and those who had cytotoxic chemotherapy, interferon therapy, or cryoablation of hepatic metastases within 2 months of study entry, were excluded, and patients who had undergone hepatic artery embolization were excluded if treatment was within 6 months, or 1 month if there were other sites of measurable disease. The required time since last dose for long-acting sst analogs, octreotide s.c., and radiolabeled sst analogs was 8 weeks, 2 days, and 6 months respectively.

\section{Study design}

This was a phase II, open-label, multicenter, and prospective study (Fig. 1). Enrolled patients selfadministered pasireotide $150 \mu \mathrm{g}$ s.c. twice daily (bid) for a 3-day test period and were assessed for achievement of partial or complete symptom control. Partial symptom control was defined as an average of $<4$ bowel movements/day, with $\leq 6$ bowel movements on any given day, and an average of $<2$ episodes of flushing/day over the time interval being studied. Complete symptom control was defined as an average of $\leq 3$ bowel movements/day for at least three consecutive days, with $\leq 3$ episodes on any given day, and no flushing episodes during the time interval being studied. If at least partial symptom control was not achieved within 3 days, the pasireotide dose was escalated by $150 \mu \mathrm{g} /$ dose up to a limit of $900 \mu \mathrm{g} /$ dose (1800 $\mu \mathrm{g}$ daily) until partial symptom control over 3 days was achieved. Dose increases up to $1200 \mu \mathrm{g}$ bid were also approved for some patients based on evidence of therapeutic benefit. If unacceptable toxicity (Common Toxicity Criteria (CTC) grade 3 or higher) occurred, a dose reduction of $150 \mu \mathrm{g}$ bid to a minimum of $150 \mu \mathrm{g}$ was permitted. Patients must have received treatment for $\geq 3$ days at any dose before further escalations were allowed.

During the study, the use of prescription medication for diarrhea was prohibited, but exceptions could have been made if the medication was required to treat an adverse event (AE). The use of dexamethasone and octreotide s.c. or other short- or long-acting sst analogs was not permitted. Patients were discouraged from taking over-the-counter medications for diarrhea. If possible, there were to be no changes in any concomitant medications. Additional treatments such as surgery or radiotherapy were prohibited. Any patients requiring these interventions were discontinued from the study before undergoing further treatment.

This study was conducted in accordance with the Declaration of Helsinki. An independent Ethics Committee or Institutional Review Board for each site approved the study protocol. All patients provided written informed consent.

\section{Assessments}

The primary efficacy outcome was symptom control (diarrhea and flushing) over any consecutive 15-day period at a fixed dose of pasireotide, assessed using a symptom diary completed daily by the patient. A physician's assessment of the patient's symptoms was performed at each scheduled visit. Patients completed the study after undergoing $\geq 15$ consecutive days with at least partial control of symptoms on a fixed dose of pasireotide but could continue to receive pasireotide as long as control was maintained and no unacceptable toxicity or evidence of disease progression was experienced. Patients who reached a dose of $900 \mu \mathrm{g}$ s.c. bid without achieving at least a partial response over a 15-day period, or were discontinued because of unacceptable drug- or disease-related AEs, were also considered to have completed the study. Pasireotide was considered effective if $30 \%$ of patients experienced at least partial symptom control on a constant dose for 15 consecutive days.

Patients were also classified as experiencing complete treatment success (responders), partial treatment success (partial responders), or treatment failure (nonresponders). Complete treatment success was defined as achieving complete symptom control based on 15 consecutive days of treatment at a constant dose level, no more than a $10 \%$ increase in biochemical parameters (serum $\mathrm{CgA}$ and urinary 5-HIAA) above baseline, and no clinical signs of disease progression as judged by the investigator. Partial treatment success was defined as achieving partial symptom control based on 15 consecutive days of treatment at a constant dose level, but without reaching complete symptom control over the same time period, no more than a $10 \%$ increase in biochemical parameters above baseline, and no significant clinical signs of disease progression, as judged by the investigator. Failure to obtain partial or complete treatment success over any consecutive 15-day period at a constant dose level was defined as treatment failure.

The secondary objective of health-related quality of life (HRQoL) was evaluated using the Functional Assessment of Chronic Illness Therapy-Diarrhea (FACIT-D), version 4. FACIT-D comprises the 
Functional Assessment of Cancer Therapy-General (FACT-G) score, which measures physical, social, emotional, and functional well-being, supplemented with a symptom-specific subscale, which measures HRQoL specific to diarrhea. All subscales were scored on a Likert scale (0-4) with higher scores indicating a better HRQoL. FACIT-D scores were obtained at baseline (day 1 immediately before pasireotide dosing) and monthly thereafter.

Tumor measurements were made using computed tomography (CT) scans or magnetic resonance imaging (MRI) at baseline and every 3 months thereafter and were assessed using RECIST version 1.0 (Therasse et al. 2000). The same method of tumor assessment used at baseline remained consistent throughout the study. Conventional CT and MRI were performed with cuts of $10 \mathrm{~mm}$ or less in slice thickness contiguously. Spiral CT was performed using a 5-8 $\mathrm{mm}$ contiguous reconstruction algorithm. The chest, abdomen, and pelvis were scanned. CT or MR images were read by the same radiologist at each assessment, accounting for all lesions that were present at baseline. All known diseases have been accounted for when assessing objective tumor status. Serum $\mathrm{CgA}$ and urinary 5-HIAA were obtained at baseline and monthly thereafter. Samples were assessed in a central laboratory.

Following 4 weeks of treatment with pasireotide, plasma samples were collected for pharmacokinetic (PK) analysis. These were collected pre-pasireotide dose and $0.5,1,2,3,4$, and $6 \mathrm{~h}$ following the morning dose of pasireotide. The PK parameters minimum plasma concentration after a single dose $\left(C_{\min }\right)$, maximum plasma concentration after a single dose $\left(C_{\max }\right)$, time to reach the maximum concentration after a single dose $\left(t_{\max }\right)$, and area under the plasma concentration-time curve from 0 to $6 \mathrm{~h}\left(\mathrm{AUC}_{0-6 \mathrm{~h}}\right)$ were assessed.

Safety and tolerability assessments consisted of reporting all AEs and serious AEs (SAEs), with severity graded according to CTC (grades 1-4). Safety and tolerability assessments were completed at screening, baseline, and monthly thereafter until study completion. Safety assessments included regular monitoring of hematology, blood chemistry, vital signs, physical condition, electrocardiogram readings, and body weight.

\section{Statistical analysis}

The total planned sample size was a minimum of 36 patients. The sample size was chosen based on the assumption of a proportion of success of 0.3 and an approximate width of 0.3 for a two-sided $95 \%$ confidence interval.

Summary statistics were provided for the primary and secondary endpoints. No formal statistical comparisons were performed for this study.

\section{Results}

Between January 2004 and August 2005, a total of 53 patients from nine centers in five countries were enrolled in the study, of whom 45 received pasireotide and were included in the safety analysis and 44 were evaluable for efficacy (Table 1). Of the 45 patients who received treatment, 36 had primary tumors of the small intestine; three had a primary tumor located in the colon, peritoneum, or lung; and five had primary tumors of unknown origin. Most patients (63.6\%) had stage IV cancer at baseline. Additionally, the majority of patients $(68.2 \%)$ were diagnosed $\geq 3$ years before enrollment into the study. At baseline, $87 \%$ of patients had liver metastases. Patients also presented with metastasis to the following locations: lymph node $(n=16$ patients), peritoneum $(n=8)$, lung $(n=5)$, bone $(n=3)$, abdomen $(n=3)$, pleura $(n=3)$, retroperitoneal $(n=2)$, pancreas $(n=2)$, kidney $(n=1)$, adrenal $(n=1)$, spleen $(n=1)$, and other $(n=3)$.

Table 1 Demographic characteristics of the safety and efficacy populations

\begin{tabular}{ll}
\hline & Safety population $(n=45)$ \\
Mean age (years (range)) & $61(40-83)$ \\
Gender $(n(\%))$ & $25(56)$ \\
Males & $20(44)$ \\
Females & \\
Race $(n(\%))$ & $44(98)$ \\
Caucasian & $1(2)$ \\
Oriental & $2(4.4)$ \\
Last dose of octreotide LAR $(n=45 ; n(\%))$ \\
10 mg & $13(29)$ \\
20 mg & $27(60)$ \\
30 mg & $1(2.2)$ \\
40 mg & $2(4.4)$ \\
Missing & Efficacy population $(n=44)$ \\
& $13(81.8)$ \\
Location of primary tumor $(n(\%))$ \\
Small intestine & $1(2.3)$ \\
Colon & $1(2.3)$ \\
Peritoneum & $1(2.3)$ \\
Lung & $5(11.4)$ \\
Unknown & $1(2.3)$ \\
Tumor stage & $0(0)$ \\
Stage I & $2(4.5)$ \\
Stage II & $28(63.6)$ \\
Stage III & $13(29.5)$ \\
Stage IV & \\
Missing & \\
\hline
\end{tabular}


Of the 45 patients who received pasireotide treatment, 17 completed the study and an additional seven continued receiving pasireotide therapy after completing the study. Discontinuation because of AEs (11 patients) was the most frequent reason for withdrawal. Eight patients withdrew their consent and new cancer therapy was prescribed for two patients. One patient was excluded from the efficacy analyzable population because no post-baseline efficacy assessments were completed. The most frequently reported concomitant medications included proton pump inhibitors $(37.8 \%)$, natural opium alkaloids (28.8\%), antipropulsives (33.3\%), and enzyme preparations $(31.1 \%)$.

\section{Symptom control with pasireotide}

Of the 44 patients included in the efficacy analyzable population, $12(27 \%)$ had complete or partial symptom control over 15 days of treatment with pasireotide. Three $(7 \%)$ of these patients achieved complete control of symptoms (two of whom received pasireotide $600 \mu \mathrm{g}$ bid and one received $900 \mu \mathrm{g}$ bid) and nine $(20 \%)$ achieved partial symptom control in response to treatment (three patients each received pasireotide 600,750 , or $900 \mu \mathrm{g}$ bid). For the 12 patients who showed complete or partial treatment response, the mean ( \pm s.D.) number of daily bowel movements was reduced from $4.3 \pm 1.4$ at baseline to $2.8 \pm 0.8$ post-pasireotide treatment, and mean ( \pm s.D.) daily flushing episodes declined from $4.9 \pm 4.1$ at baseline to $1.0 \pm 0.8$ post-treatment (Fig. 2 ). The mean duration of complete and partial symptom control was 43.7 (range 26-58) days and 72.0 (range 15-244) days respectively. The median treatment duration was 91 (range 1-595) days.
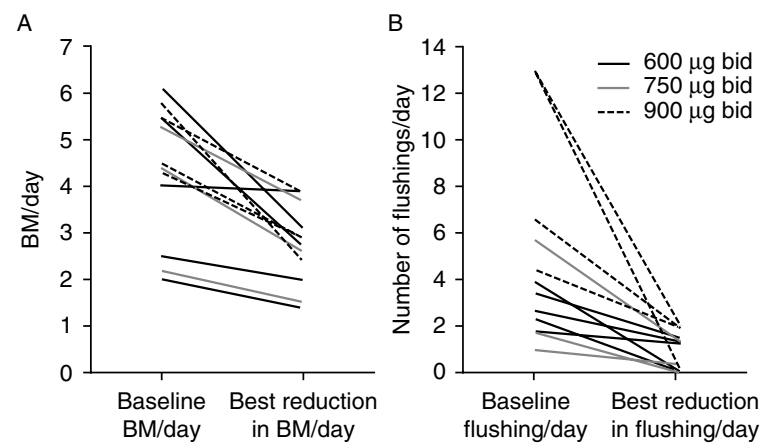

Figure 2 Clinical symptom responses following pasireotide treatment in responding patients: $(A)$ reductions in the number of bowel movements (BM) per day from baseline and $(B)$ reductions in the number of flushing episodes per day from baseline. Patients received pasireotide 600,750 , or $900 \mu$ g s.c. bid.
Table 2 Mean physical well-being score over time in responders, nonresponders, and patients who discontinued before 3 months of therapy with pasireotide

\begin{tabular}{|c|c|c|c|c|}
\hline & Baseline & Month 1 & Month 2 & Month 3 \\
\hline \multicolumn{5}{|c|}{ Responders by month 3} \\
\hline$n$ & 12 & 12 & 10 & 11 \\
\hline $\begin{array}{l}\text { Mean score } \\
\text { (S.D.) }\end{array}$ & $21.1(3.7)$ & $21.4(6.3)$ & $20.0(4.5)$ & $21.3(3.5)$ \\
\hline Range & $15.8-27.0$ & $8.0-28.0$ & $11.2-26.0$ & $16.0-26.0$ \\
\hline \multicolumn{5}{|c|}{ Nonresponders by month 3} \\
\hline$n$ & 13 & 13 & 13 & 13 \\
\hline $\begin{array}{l}\text { Mean score } \\
\text { (S.D.) }\end{array}$ & $21.0(3.1)$ & $21.4(4.5)$ & $21.1(4.6)$ & $22.3(3.7)$ \\
\hline Range & $16.0-26.0$ & $11.0-27.0$ & $13.0-27.0$ & $17.0-27.0$ \\
\hline \multicolumn{5}{|c|}{ Patients who discontinued before 3 months of therapy } \\
\hline$n$ & 19 & 15 & 8 & - \\
\hline $\begin{array}{l}\text { Mean score } \\
\text { (S.D.) }\end{array}$ & $18.0(5.5)$ & $17.8(6.1)$ & $15.8(5.8)$ & - \\
\hline Range & $9.0-27.0$ & $5.0-27.0$ & $5.0-24.0$ & - \\
\hline
\end{tabular}

When assessing the number of patients with complete treatment success, partial treatment success, and treatment failure, one (2.3\%) patient achieved complete treatment success and four $(9.1 \%)$ patients achieved partial treatment success. The remaining 39 (88.6\%) patients were classified as treatment failures.

\section{Tumor response}

Overall tumor response based on RECIST following treatment with pasireotide was assessed in 24 of the 44 patients. At the last assessment before study exit, $57 \%(13 / 23)$ of patients had stable disease, $43 \%(10 / 23)$ of patients had progressive disease, and one patient (4\%) had an unknown response. There were no complete or partial tumor responses observed.

\section{Health-related quality of life}

Of the 45 patients who received treatment, 30 were included in the HRQoL analysis. In the clinical responder group, more patients had mean HRQoL scores for functional and physical well-being, symptom-specific scores, and FACIT-D summary scores that were slightly higher or the same scores at the end of treatment than at baseline, suggesting an improvement in HRQoL. In the nonresponder group, similar numbers of patients recorded an improvement or a worsening in HRQoL (Table 2).

\section{Biochemical marker data}

Median percentage changes in 5-HIAA and CgA levels from baseline in patients with evaluable data are depicted in Fig. 3. Levels of both markers decreased rapidly after pasireotide initiation and remained low. 


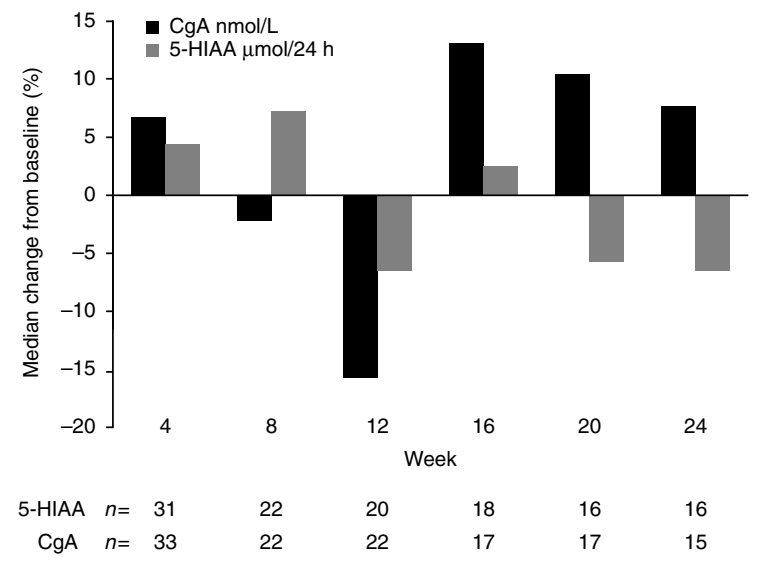

Figure 3 Median percentage change in 5-HIAA and CgA levels from baseline in patients with evaluable data.

\section{Pharmacokinetics}

Thirty-five of the 37 patients with available PK plasma samples were analyzed; one patient was excluded because of early discontinuation from the study and one patient was excluded because of missing pre-dose plasma PK samples. The plasma concentration vs time profiles are shown in Fig. 4 and the PK parameters of $C_{\min }, C_{\max }, t_{\max }$, and $\mathrm{AUC}_{0-6 \mathrm{~h}}$ for pasireotide are reported in Table 3. Exposure to pasireotide appeared to increase dose proportionally with pasireotide treatment from 150 to $1200 \mu \mathrm{g}$ bid, as determined by the mean values for $C_{\max }, C_{\min }$, and $\mathrm{AUC}_{0-6 \mathrm{~h}}$ (Table 3).

\section{Safety and tolerability}

Forty-four of the 45 patients (98\%) included in the safety population experienced one or more $\mathrm{AE}$, with 36 patients $(80 \%)$ considered to have study drugrelated AEs (Table 4). The most common AEs with a suspected study drug relationship were nausea (27\%), abdominal pain (20\%), weight loss (20\%), and hyperglycemia $(16 \%)$ and most were of mild or moderate severity (CTC grades 1 (36\%) and $2(24 \%))$. Fourteen patients $(31 \%)$ experienced SAEs; however, only one of these events was suspected to be study drug related; this patient had a CTC grade 4 increase in lipase, which resolved spontaneously and no action was taken. One patient died during the study because of tumor progression that was not considered study drug related. AEs that led to patient discontinuations were general physical health deterioration (grade 3, suspected drug relationship), nausea (grade 3, suspected drug relationship), tumor progression (grade 4, unrelated to study drug), diarrhea (grade 3, unrelated to study drug), abdominal pain (grade 3, suspected drug relationship), bone pain due to metastasis (grade 4, unrelated to study drug), abdominal cramping and bloating (each grade 2, both suspected drug relationship), tumor metastasis (grade 3, unrelated to study drug), worsening diarrhea (grade 2, suspected drug relationship), worsening hyperglycemia (grade 3, suspected drug relationship), dry heaves, nausea and vomiting (each grade 1, all unrelated to study drug), hypotension, and increased flushing (grades 2 and 1 respectively; both not related to study drug).

Weight loss occurred in 19 patients (42\%) during the study, all cases of which were grades 1 or 2 , with nine of the cases (20\%) considered related to pasireotide. Maximum mean weight loss occurred within days 112-196 (weeks 16-28) of treatment with pasireotide, with a stabilization of effect after approximately week 28. There was no apparent relationship between weight loss and pasireotide dose.

Twenty-five patients were assessed for changes in blood glucose levels. Of these, 15 had baseline fasting blood glucose (FBG) levels $<100 \mathrm{mg} / \mathrm{dl}$. At the last assessment before study exit, four of the 15 patients continued to have FBG $<100 \mathrm{mg} / \mathrm{dl}$; five of the 15 patients had the highest post-baseline blood glucose level fall into the $100-126 \mathrm{mg} / \mathrm{dl}$ range and six had the highest post-baseline level fall into $>126 \mathrm{mg} / \mathrm{dl}$. Seven patients had baseline FBG levels between 100 and $126 \mathrm{mg} / \mathrm{dl}$; of these patients, one remained in the $100-126 \mathrm{mg} / \mathrm{dl}$ range and six had the highest postbaseline level increased to $>126 \mathrm{mg} / \mathrm{dl}$. The three patients with baseline FBG $>126 \mathrm{mg} / \mathrm{dl}$ remained in this category during treatment. For the majority of patients, the effect on FBG was transient as a result of

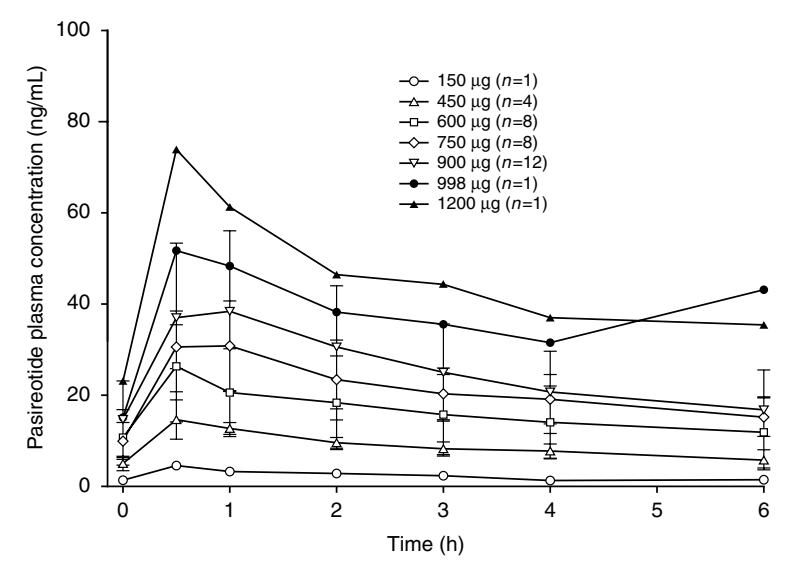

Figure 4 Plasma concentration (mean \pm s.D.) vs time profiles of pasireotide following 28 days of multiple bid pasireotide dosing. 
Table 3 Pharmacokinetic parameters of pasireotide on day 28 following multiple bid dosing of pasireotide

\begin{tabular}{lccccc}
\hline Dose $(\mu \mathrm{g})$ & $\boldsymbol{n}$ & $\boldsymbol{t}_{\max }(\mathrm{h})$ & $\boldsymbol{C}_{\max }(\mathrm{ng} / \mathrm{ml})$ & $\boldsymbol{C}_{\min }(\mathrm{ng} / \mathrm{ml})$ & AUC $_{\mathbf{0}-6 \mathbf{h}}(\mathrm{ng} / \mathrm{h} \mathrm{per} \mathrm{ml)}$ \\
\hline 150 & 1 & 0.50 & 4.7 & 1.4 & 15.0 \\
450 & 4 & $0.79(0.50-1.00)$ & $15.2 \pm 3.9$ & $5.1 \pm 1.6$ & $58.4 \pm 9.3$ \\
600 & 8 & $0.50(0-0.50)$ & $26.4 \pm 12.2$ & $9.9 \pm 6.4$ & $105 \pm 53$ \\
750 & 8 & $0.75(0.50-1.17)$ & $31.6 \pm 9.8$ & $10.0 \pm 4.0$ & $128 \pm 36$ \\
900 & 12 & $1.00(0.50-3.00)$ & $40.6 \pm 17.8$ & $13.5 \pm 7.1$ & $155 \pm 66$ \\
998 & 1 & 0.52 & 51.9 & 23.3 & 231 \\
1200 & 1 & 0.50 & 73.8 & 278 \\
\hline
\end{tabular}

Data are described as median (range) for $t_{\max }$ and mean \pm s.D. for all others. For groups with fewer than three patients, only median or mean values are reported. $\mathrm{AUC}_{0-6 \mathrm{~h}}$, area under the plasma concentration-time curve from 0 to $6 \mathrm{~h} ; C_{\max }$, maximum plasma concentration after a single dose; $C_{\min }$, minimum plasma concentration after a single dose; $t_{\max }$, time to reach the maximum concentration after a single dose.

adjustments to the dose of pasireotide and appropriate antidiabetic management. Three of four patients who experienced an $\mathrm{AE}$ of worsening diabetic symptoms had a medical history of active diabetes mellitus, and three of the seven patients who experienced hyperglycemia had a history of active hyperglycemia at baseline. One additional patient who experienced an AE of hyperglycemia had a history of diabetes mellitus at baseline.

\section{Discussion}

Pasireotide 600-900 $\mu \mathrm{g}$ s.c. bid was effective in controlling the symptoms of diarrhea and flushing in $27 \%$ of patients with advanced NETs who were no longer responsive to octreotide LAR therapy. No clear dose-response relationship with regard to symptom control was observed. There was a minimal difference between the defined symptom reduction success rate of $30 \%$ and the observed $27 \%$ reduction of symptoms. Although the study did not meet its primary efficacy endpoint, potential activity of pasireotide in patients with advanced NETs refractory or resistant to octreotide LAR was observed and further studies are warranted When observing HRQoL, the functional and physical well-being, symptom-specific scores, and total FACIT-D assessments showed some variability. Although there were no clear trends between patients who responded to pasireotide treatment and HRQoL, these results suggest that patients who responded to pasireotide improved their HRQoL slightly.

Recently, the antiproliferative effect of octreotide LAR was demonstrated in the randomized, doubleblind, placebo-controlled PROMID study in patients with advanced, intestinal (midgut) NETs (Rinke et al. 2009), in which octreotide LAR $30 \mathrm{mg} / 28$ days led to a significant increase in time to tumor progression compared with placebo (14.3 vs 6.0 months; $P=0.000072$ ), regardless of whether patients had a functioning or nonfunctioning tumor (Arnold et al. 2009, Rinke et al. 2009). In line with the antitumor effects of octreotide, pasireotide has been shown to have direct and indirect antitumor effects in vitro, including sst receptor-mediated apoptosis and inhibition of cell proliferation (Adams et al. 2004, Lattuada et al. 2007, Ono et al. 2007). In the current study, pasireotide treatment resulted in stable disease in more than half of the patients, which is a clinically relevant achievement in patients with functioning NETs refractory or resistant to octreotide LAR.

Pasireotide was absorbed rapidly, with $C_{\max }$ observed within $1 \mathrm{~h}$ post-dose in the majority of cases. Pasireotide exposure, assessed by $C_{\min }, C_{\max }$, and $\mathrm{AUC}_{0-6 \mathrm{~h}}$, appeared to be dose proportional; however, this effect could not be confirmed as formal statistical analysis was not conducted. An increased pasireotide concentration was observed at $6 \mathrm{~h}$ in the patient administered pasireotide $998 \mu \mathrm{g}$ bid. As pasireotide has a high metabolic stability and does not

Table 4 Most frequently reported study drug-related adverse events for any dose group $\geq 5 \%{ }^{\text {a }}(n=45)$

\begin{tabular}{lr}
\hline Adverse event & $\boldsymbol{n}(\%)$ \\
\hline Nausea & $12(26.7)$ \\
Abdominal pain & $9(20.0)$ \\
Weight decrease & $9(20.0)$ \\
Hyperglycemia & $7(15.6)$ \\
Diabetes mellitus & $4(8.9)$ \\
Dysgeusia & $4(8.9)$ \\
Flatulence & $4(8.9)$ \\
Fatigue & $4(8.9)$ \\
Asthenia & $3(6.7)$ \\
Vertigo & $3(6.7)$ \\
Diarrhea & $3(6.7)$ \\
Headache & $3(6.7)$ \\
\hline
\end{tabular}

${ }^{a}$ Grade 3 adverse events of any frequency included diabetes mellitus $(n=1)$, hyperglycemia $(n=3)$, abdominal pain $(n=2)$, vomiting $(n=1)$, general physical health deterioration $(n=1)$, procedural nausea $(n=1)$, and blood amylase increase $(n=1)$. One patient experienced a grade 4 increase in lipase levels. 
interact with CYP450 enzymes or P-glycoprotein, a potential drug-drug interaction is unlikely, especially as the increase was not correlated with the presence of a concomitant medication. As the increased pasireotide concentration was observed at one time point only, the finding can be attributed to bioanalytical assay variability. In the patients who responded to pasireotide treatment, the median effective plasma concentration of pasireotide was observed at around $10 \mathrm{ng} / \mathrm{ml}$, which was based on the observed mean value of $C_{\min }$ at pasireotide 600, 750, and $900 \mu \mathrm{g}$ bid. At these three dose levels, clinical response (symptom control) to pasireotide was observed with similar frequency.

There is a well-established safety profile for sst analogs, attributable to their clinical use in the treatment for NETs for over two decades. The most frequent AEs are abdominal pain with cramps, constipation, diarrhea, steatorrhea, injection-site irritation, nausea, and vomiting (Modlin et al. 2010). In the current study, a similar tolerability profile was observed, with most AEs being of mild or moderate severity. Some patients required an adjustment to the dose of pasireotide, and additional therapy was prescribed to treat some of the AEs. Treatment with pasireotide at all doses examined in this study was associated with hyperglycemia. Hyperglycemia has previously been observed in up to $27 \%$ of patients administered long-acting sst analogs (Ipsen Ltd. Somatuline depot (lanreotide) injection prescribing information 2007, available at: www.somatulinedepot. com/hcp/full-prescribing-information.asp; Novartis Sandostatin LAR Depot (octreotide acetate for injectable suspension) prescribing information 2010, available at: www.pharma.us.novartis.com/product/pi/pdf/ sandostatin_lar.pdf). Impaired glucose tolerance or even overt diabetes mellitus has been seen in select cases (Oberg 2004). For the majority of patients in the current study, hyperglycemia was transitory as adjustments were made to pasireotide dosing, oral antidiabetic treatment, and/or diet. Increased FBG was more evident in patients with a history of impaired FBG or diabetes mellitus. A slightly higher incidence of AEs was reported with the 750 and $900 \mu \mathrm{g}$ bid dose groups, but no overall clear dose dependence was observed. Studies in other patient populations have demonstrated that pasireotide does not affect glucagon release. Changes in glucose metabolism are most obvious following initial treatment and appear less pronounced with ongoing therapy (Boscaro et al. 2009).

Nineteen cases of weight loss followed by stabilization were reported during the study. Studies in patients with NETs administered octreotide or lanreotide have found that $\sim 5-15 \%$ of patients may experience weight decrease during treatment, although these events have not been confirmed as drug related (Ipsen Ltd. Somatuline depot (lanreotide) injection prescribing information 2007, available at: www. somatulinedepot.com/hcp/full-prescribing-information. asp; Novartis Sandostatin LAR Depot (octreotide acetate for injectable suspension) prescribing information 2010, available at: www.pharma.us.novartis. com/product/pi/pdf/sandostatin_lar.pdf). In general, few studies report details of weight change in patients with NETs.

The response rate seen in this study may be attributed to the fact that difficult-to-treat patients were evaluated. Further studies may identify subgroups with greater rates of tumor response. Pasireotide may potentially be more effective in early treatment of patients with well-differentiated tumors. Additionally, although the majority of patients appeared to experience stable disease during treatment with pasireotide, interpretation of this finding is complex. Randomized studies, such as the PROMID trial that confirmed the antitumor effect of octreotide LAR (Rinke et al. 2009), are required to confirm any such effect with pasireotide. A phase III study evaluating pasireotide LAR vs octreotide LAR is ongoing in patients with metastatic carcinoid NETs whose disease-related symptoms are inadequately controlled by sst analogs.

In conclusion, clinical responses to pasireotide treatment were seen at doses of $600 \mu \mathrm{g}$ bid and higher, and pasireotide controlled the symptoms of diarrhea and flushing in $27 \%$ of patients with advanced NETs who were refractory or resistant to the standard therapy, octreotide LAR. HRQoL was maintained with pasireotide therapy. Pasireotide treatment was generally well tolerated, with adverse gastrointestinal events being the most common. These results demonstrate that pasireotide is a potential treatment option for patients with symptoms of carcinoid syndrome who are resistant or refractory to octreotide LAR therapy. A phase III study comparing pasireotide LAR with octreotide LAR in patients with advanced NETs is ongoing.

\section{Declaration of interest}

L K Kvols has consulted, received research funding and honoraria from Novartis, and has also received research funding from Pfizer, Lexicon Pharmaceutical, and the Southwest Oncology Group (SWOG). K E Oberg has received honoraria from Novartis, Ipsen, and Pfizer and serves on advisory boards for Novartis, Ipsen, and Pfizer. T M O'Dorisio has nothing to disclose. W W de Herder has consulted for Novartis Pharmaceuticals. R Arnold has received research funding and honoraria from Novartis. L Anthony has served on advisory boards for Novartis. 
B Wiedenmann has received consulting and lecture fees and payment for educational presentations from Pfizer. P Mohideen, K Hu, Y Zhang, and G Hughes are employees of Novartis.

\section{Funding}

Financial support for medical editorial assistance was provided by Novartis Pharmaceuticals Corporation. This study was funded by Novartis Pharma AG.

\section{Author contribution statement}

L K Kvols, K E Oberg, T M O’Dorisio, W W de Herder, R Arnold, L Anthony, and B Wiedenmann contributed to the design and conduct of the study, as well as acquisition and interpretation of data. P Mohideen, K Hu, Y Zhang, and $G$ Hughes contributed to the design of the study and interpretation of data. G Hughes performed the statistical analysis of data. All authors critically reviewed and approved the final manuscript.

\section{Acknowledgements}

The authors acknowledge the following investigators for their participation in this study: Anthony Heaney and Philippe Ruszniewski. The authors thank Keri Wellington and Helene Darmofal for medical editorial assistance with this manuscript.

\section{References}

Adams RL, Adams IP, Lindow SW \& Atkin SL 2004 Inhibition of endothelial proliferation by the somatostatin analogue SOM230. Clinical Endocrinology 61 431-436. (doi:10.1111/j.1365-2265.2004.02098.x)

Arnold R, Muller H, Schade-Brittinger C, Rinke A, Klose K, Barth P, Wied M, Mayer C, Aminossadati B \& PROMID Study Group 2009 Placebo-controlled, double-blind, prospective, randomized study of the effect of octreotide LAR in the control of tumor growth in patients with metastatic neuroendocrine midgut tumors: a report from the PROMID study group. Journal of Clinical Oncology 27 (15S) abstract 4508.

Boerlin V, Van Der Hoek J, Beglinger C, Poon KW, Hartmann S, Dutreix C, Kovarik JM, Bruns C, Weckbecker G, Lewis I et al. 2003 New insights on SOM230, a universal somatostatin receptor ligand. Journal of Endocrinological Investigation 26 (8 Suppl) 14-16.

Boscaro M, Ludlam WH, Atkinson B, Glusman JE, Petersenn S, Reincke M, Snyder P, Tabarin A, Biller BM, Findling J et al. 2009 Treatment of pituitary dependent Cushing's disease with the multi-receptor ligand somatostatin analog pasireotide (SOM230): a multicenter, phase II trial. Journal of Clinical Endocrinology and Metabolism 94 115-122. (doi:10.1210/jc.2008-1008)
Bruns C, Weckbecker G, Raulf F, Kaupmann K, Schoeffter P, Hoyer D \& Lubbert H 1994 Molecular pharmacology of somatostatin-receptor subtypes. Annals of the New York Academy of Sciences 733 138-146. (doi:10.1111/j.17496632.1994.tb17263.x)

Bruns C, Lewis I, Briner U, Meno-Tetang G \& Weckbecker G 2002 SOM230: a novel somatostatin peptidomimetic with broad somatotropin release inhibiting factor (SRIF) receptor binding and a unique antisecretory profile. European Journal of Endocrinology 146 707-716. (doi:10.1530/eje.0.1460707)

Fajans SS \& Vinik AI 1989 Insulin-producing islet cell tumors. Endocrinology and Metabolism Clinics of North America 18 45-74.

Hofland LJ \& Lamberts SW 2003 The pathophysiological consequences of somatostatin receptor internalization and resistance. Endocrine Reviews 24 28-47. (doi:10.1210/er. 2000-0001)

Hofland LJ, Van Der Hoek J, Feelders R, van der Lely AJ, De Herder W \& Lamberts SW 2005 Pre-clinical and clinical experiences with novel somatostatin ligands: advantages, disadvantages and new prospects. Journal of Endocrinological Investigation 28 (11 Suppl) 36-42.

Lattuada D, Casnici C, Crotta K, Mastrotto C, Franco P, Schmid HA \& Marelli O 2007 Inhibitory effect of pasireotide and octreotide on lymphocyte activation. Journal of Neuroimmunology 182 153-159. (doi:10.1016/ j.jneuroim.2006.10.007)

Li M, Li W, Kim HJ, Yao Q, Chen C \& Fisher WE 2004 Characterization of somatostatin receptor expression in human pancreatic cancer using real-time RT-PCR. Journal of Surgical Research 119 130-137. (doi:10.1016/ j.jss.2004.03.006)

Loftus JP \& van Heerden JA 1995 Surgical management of gastrointestinal carcinoid tumors. Advances in Surgery 28 317-336.

Long RG, Bryant MG, Mitchell SJ, Adrian TE, Polak JM \& Bloom SR 1981 Clinicopathological study of pancreatic and ganglioneuroblastoma tumours secreting vasoactive intestinal polypeptide (vipomas). BMJ 282 1767-1771. (doi:10.1136/bmj.282.6278.1767)

Modlin IM, Kidd M, Latich I, Zikusoka MN \& Shapiro MD 2005 Current status of gastrointestinal carcinoids. Gastroenterology 128 1717-1751. (doi:10.1053/j.gastro. 2005.03.038)

Modlin IM, Latich I, Kidd M, Zikusoka M \& Eick G 2006 Therapeutic options for gastrointestinal carcinoids. Clinical Gastroenterology and Hepatology 4 526-547. (doi:10.1016/j.cgh.2005.12.008)

Modlin IM, Pavel M, Kidd M \& Gustafsson BI 2010 Review article: somatostatin analogs in the treatment of gastro-entero-pancreatic neuroendocrine (carcinoid) tumors. Alimentary Pharmacology \& Therapeutics 31 169-188. (doi:10.1111/j.1365-2036.2009.04174.x)

Oberg K 2004 Management of neuroendocrine tumors. Annals of Oncology 15 iv293-iv298. (doi:10.1093/ annonc/mdh216) 
Ono K, Suzuki T, Miki Y, Taniyama Y, Nakamura Y, Noda Y, Watanabe M \& Sasano H 2007 Somatostatin receptor subtypes in human non-functioning neuroendocrine tumors and effects of somatostatin analogue SOM230 on cell proliferation in cell line NCIH727. Anticancer Research 27 2231-2239.

Plockinger U, Rindi G, Arnold R, Eriksson B, Krenning EP, de Herder WW, Goede A, Caplin M, Oberg K, Reubi JC et al. 2004 Guidelines for the diagnosis and treatment of neuroendocrine gastrointestinal tumours. A consensus statement on behalf of the European Neuroendocrine Tumour Society (ENETS). Neuroendocrinology 80 394-424. (doi:10.1159/000085237)

Rinke A, Müller H-H, Schade-Brittinger C, Klose K-J, Barth P, Wied M, Mayer C, Aminossadati B, Pape U-F, Bläker M et al. 2009 Placebo-controlled, double-blind, prospective, randomized study of the effect of octreotide LAR in the control of tumor growth in patients with metastatic neuroendocrine midgut tumors: a report from the PROMID study group. Journal of Clinical Oncology 27 4656-4663. (doi:10.1200/JCO.2009.22.8510)

Ronga G, Salerno G, Procaccini E, Mauro L, Annovazzi A, Barone R, Mellozzi M, Tamburrano G \& Signore A 1995 111In-octreotide scintigraphy in metastatic medullary thyroid carcinoma before and after octreotide therapy: in vivo evidence of the possible down-regulation of somatostatin receptors. Quarterly Journal of Nuclear Medicine 39 134-136.
Rubin J, Ajani J, Schirmer W, Venook AP, Bukowski R, Pommier R, Saltz L, Dandona P \& Anthony L 1999 Octreotide acetate long-acting formulation versus openlabel subcutaneous octreotide acetate in malignant carcinoid syndrome. Journal of Clinical Oncology 17 600-606.

Schmid HA \& Schoeffter P 2004 Functional activity of the multiligand analog SOM230 at human recombinant somatostatin receptor subtypes supports its usefulness in neuroendocrine tumors. Neuroendocrinology 80 (Suppl 1) 47-50. (doi:10.1159/000080741)

Therasse P, Arbuck SG, Eisenhauer EA, Wanders J, Kaplan RS, Rubinstein L, Verweij J, Van Glabbeke M, van Oosterom AT, Christian MC et al. $2000 \mathrm{New}$ guidelines to evaluate the response to treatment in solid tumors. European Organization for Research and Treatment of Cancer, National Cancer Institute of the United States, National Cancer Institute of Canada. Journal of the National Cancer Institute 92 205-216. (doi:10.1093/jnci/ 92.3.205)

Received in final form 12 June 2012

Accepted 27 June 2012

Made available online as an Accepted Preprint 17 July 2012 